\title{
Modelo de Naciones Unidas: una herramienta constructivista
}

\section{Model United Nations: a constructivist tool}

\author{
Fernando David Márquez Duarte es maestro en Desarrollo Regional por el Colegio de la Frontera \\ Norte (COLEF) (fdmdj@hotmail.com) (D) (https://orcid.org/0000-0001-5653-5002)
}

Recibido: 2019-01-07 / Revisado: 2019-03-21 / Aceptado: 2019-05-27 / Publicado: 2019-07-01

\section{Resumen}

En el presente documento se presenta la investigación realizada sobre el caso de estudio del Modelo de Naciones Unidas (MUN) en un grupo de estudiantes de la Maestría en Educación: Campo Educación ambiental (MECEA) de la Universidad Pedagógica Nacional (UPN) Mexicali. Esta investigación presenta relevancia para el campo de estudio de la educación, específicamente desde un enfoque constructivista, así como desde la pedagogía crítica, ya que se analiza la pertinencia de los MUNs como herramienta pedagógica para fomentar conocimientos y capacidades en los educandos, desarrollando una conciencia crítica. La investigación realizada ha sido conducida enteramente con un enfoque cualitativo de caso de estudio con un método etnográfico, incluyendo instrumentos como la observación participativa, entrevistas semi-estructuradas y diario de campo. Las principales conclusiones a las que se llegaron son que en el caso de estudio los MUNs han mostrado ser una herramienta pedagógica útil para fomentar y poner en práctica conocimientos sobre educación ambiental, impacto ambiental, cooperación internacional, la agenda 2030, entre otros, así como capacidades necesarias para un educador ambiental como la negociación, el hablar en público, el lenguaje formal y el debate. Aspectos del MUN como el juego de rol, la Zona de Desarrollo Próximo (ZDP) y las interacciones entre participantes dentro de un marco diplomá- tico han mostrado ser cruciales para los resultados que estos ejercicios fomentan en sus participantes.

Descriptores:Educación, constructivismo, MUN, capacidades, educación ambiental, pedagogía.

\begin{abstract}
In the following document, the research about the study case of the Model United Nations (MUN) in a group of students of the Master's Degree in Education: Environment Education Field (MECEA) of the National Pedagogy University (UPN) Mexicali is presented. This research is relevant for the education study field, specifically from a constructivist approach and from the critical pedagogy, because it is analyzed the importance of MUN as a pedagogic tool to foster knowledge and capabilities in the students, developing a critical consciousness. The research has been conducted entirely with a qualitative focus of study case with an ethnographic method, including instruments as participatory observation, semi-structured interviews and field journal. The main findings reached are that in the study case analyzed, MUNs have shown to be a useful pedagogic tool to foster and practice knowledge about environmental education, environmental impact, international cooperation, the 2030 agenda, as well as necessary capabilities for an environmental educator, such as negotiation, public speaking, formal
\end{abstract}

Forma sugerida de citar: Márquez Duarte, F. (2019). Modelo de Naciones Unidas: una herramienta constructivista. Alteridad, 14(2), 267-278. https://doi.org/10.17163/alt.v14n2.2019.10 
language and debate. Aspects of the MUN like roleplaying, Zone of Proximal Development (ZPD) and the interactions between participants in a diplomatic

\section{Introducción y estado de la cuestión}

En estudios pedagógicos, una de las grandes propuestas teóricas es la constructivista. En un panorama actual, esta teoría se ha convertido en central en diferentes modelos educativos alrededor del mundo. En este documento se presenta el resultado del uso del Modelo de Naciones Unidas (MUN) como herramienta constructivista para el aprendizaje y la puesta en práctica de conocimientos y capacidades en un grupo de alumnos de la Maestría en Educación: Campo Educación ambiental (MECEA) de la Universidad Pedagógica Nacional (UPN) en Mexicali, Baja California (BC), México.

Al ser realizada esta investigación en un marco de posgrado de educación ambiental, es importante considerar el concepto de la educación ambiental, que se entiende como:

La acción educativa por la cual la comunidad educativa tiende a la toma de conciencia de su realidad global, del tipo de relaciones que los hombres establecen entre sí y con la naturaleza, de los problemas derivados de dichas relaciones y sus causas profundas... (Tamayo citado Aguirre Ibarra \& Mata González, 2011, p. 61).

Otros aspectos centrales en la educación ambiental son fomentar en los ciudadanos conciencia intercultural, conciencia global y ser agentes de cambio social colectivo (Mata González, \& Aguirre Ibarra, 2007).

La discusión sobre los postulados y la esencia del constructivismo que se discuten en este documento, parten de las propuestas de Vygotsky principalmente, así como de Piaget en segundo plano. Por otro lado, los postulados de la pedagogía crítica se analizan a partir de los postulados de Freire.

El constructivismo propone que las personas crean su propio aprendizaje, de manera frame have proven to be crucial for the results that these exercises foster in the participants.

Keywords: Education, constructivism, MUN, capabilities, environmental education, pedagogy.

que el conocimiento no es impuesto desde el exterior del individuo sino que se forma dentro de ellos. En el constructivismo los estudiantes deben estructurar situaciones donde participen de manera activa a través de la interacción social (Schunk, 1997).

Los constructivistas declaran que el pensamiento se lleva a cabo en situaciones (que Piaget denomina conflictos cognoscitivos) y que las personas construyen el conocimiento con base en sus experiencias en estas situaciones (Bredo citado en Schunk, 1997). Según esta teoría, la motivación depende de la actividad cognoscitiva al interactuar con factores sociales y las formas de la enseñanza, incluyendo medios de apoyo de la misma y lenguaje (Sivan citado en Schunk, 1997).

Si bien Piaget y Vygotsky tienen ideas que se contraponen, convergen en la esencia del modelo constructivista, donde el alumno es el que descubre un abanico de conocimientos, capacidades y oportunidades, fomentado por la guía del profesor, por medio se situaciones o controversias.

Para Piaget, el aprendizaje se hace tangible cuando los educandos experimentan un conflicto cognoscitivo y lo asimilan para construir o modificar estructuras internas. El pedagogo argumentaba que era necesario provocar controversias para que el educando obtuviera aprendizaje, ya que para él, el desarrollo sucede cuando las estructuras cognoscitivas del educando chocan con los estímulos de fuera; esta situación se fomenta mediante el planteamiento de problemas a alumnos, para que estos puedan resolverlos (Schunk, 1997). Es importante resaltar que al plantear problemas a alumnos se ponen en práctica conocimientos y habilidades que han sido previamente transmitidos por el profesor. Así mismo Piaget plantea la necesidad 
de la interacción social, para que el educando comprenda que existen diferentes puntos de vista, haciendo que se vuelvan menos egocéntricos (Schunk, 1997).

Por su parte, Vygotsky argumenta que la interacción de factores sociales, históricocontextuales e individuales da como resultado el desarrollo del individuo. Para Vygotsky, la importancia de las interacciones radica en que permiten a los individuos transformar sus experiencias basándose en sus conocimientos, para reestructurar el conocimiento que obtienen y que previamente tenían. Vygotsky sostiene que es imposible separar el aprendizaje y el desarrollo del contexto en el que se insertan (Schunk, 1997). Este postulado tiene una relación cercana con los postulados de Freire (1970), ya que para el brasileiro, educar es crear una situación en donde el individuo aprenda a tomar consciencia de su entorno por sí mismo, con el apoyo del educador que funge como guía. De manera que Vygotsky y Freire convergen en cuanto a que el educando mediante las interacciones con su entorno es el que debe tomar conciencia y aprendizaje; el educador con base en estos postulados funciona como guía, no impone conocimientos, sino que apoya a que el educando logre tomar conciencia de su realidad conforme a su percepción, misma que para ambos autores debe ser crítica.

Uno de los principales argumentos de Vygotsky es que el conocimiento se construye mediante la internalización de las acciones y procesos mentales que suceden por medio de las interacciones sociales (Schunk, 1997). Este postulado es sumamente relevante para este trabajo, ya que en los MUN (que se explicarán más adelante), las interacciones sociales tienen un papel crucial en los aprendizajes y capacidades practicados y generados en el ejercicio. En esta línea es importante retomar los postulados de la educación liberadora propuesta por Freire (1970), la cual el pedagogo presenta como una educación problematizadora, que busca generar conciencia en los educandos, siendo el diálogo un elemento central para crear conciencia, estimulando la reflexión sobre las coyunturas y procesos históricos que nos han llevado a estas coyunturas. Este postulado también refuerza lo que Piaget propone en un modelo constructivista; que se presenten a los alumnos situaciones o controversias que los lleven a generar conciencia con el objetivo de lograr un aprendizaje más integral, de una manera más práctica.

Uno de los aspectos centrales para este documento sobre el modelo constructivista es la Zona de Desarrollo Próximo (ZDP), este concepto es analizado ampliamente en los trabajos de Vygotsky y se discute a continuación, incluyendo la importancia del mismo para el caso de estudio de esta investigación. La ZDP se puede definir como la zona que comprende el rango o espectro entre el nivel actual del desarrollo de un individuo, determinada mediante la solución independiente de problemas, y el nivel de desarrollo que puede alcanzar (potencial), determinado por medio de la solución de problemas con el apoyo de un guía, pudiendo ser el profesor o individuos con mayor nivel de capacidad (Vygotsky citado en Schunk, 1997).

El educando internaliza el conocimiento y/o capacidades debido a las interacciones con otros individuos en las que forma parte, creando un cambio cognoscitivo. En la ZDP los educandos utilizan su propia percepción y entendimiento de las interacciones sociales para traducir estas experiencias a significados, integrando una comprensión de las situaciones, misma que les permite realizar tareas que de manera independiente no podían realizar.

En una ZDP, los individuos participantes (en un contexto determinado) obtienen mayor aprendizaje, conocimiento y/o capacidades por el estímulo y apoyo de las interacciones con los demás participantes (Kempston, \& Thomas, 2014). Como se puede analizar, una parte crucial de la ZDP es lo referente a las interacciones sociales; los postulados constructivistas de Vygotsky tienen a este concepto en una consideración central, ya que las interacciones son 
las que permiten que los individuos logren un mayor desarrollo como individuos.

Continuando con la discusión de postulados teóricos relevantes para el caso de estudio de este documento, es necesario retomar los postulados de Freire (1970). El pedagogo brasileiro propone una pedagogía de la concientização (concientización), que debe utilizar un método crítico y dialógico y debe usar técnicas innovadoras para lograr que el individuo llegue a la meta de la pedagoga crítica, que según Freire es la emancipación. El desarrollar una conciencia crítica implica participación política para el brasileiro, ya que implica una capacidad de decisión y compromiso con la sociedad. Como se puede apreciar, los postulados de la pedagogía crítica tienen un fuerte enfoque colectivo, buscando que los educandos busquen el beneficio colectivo y no el individual, por lo que el aspecto dialógico se torna aún más importante. En esta misma línea, Freire (1970) considera métodos activos para generar una concientización en los educandos, donde se desarrolle una capacidad crítica en los individuos a través del debate colectivo, mediante situaciones desafiantes que se plantean por el educador. Esta interacción se propone como una relación dialógica, no de imposición de ideas; una relación horizontal de discusión de ideas para llegar a reflexiones más integrales e inclusivas.

Aunando sobre el desarrollo de una conciencia crítica, se propone que ésta comienza con una conciencia interior de cada individuo; de su esencia como producto de un proceso histórico desarrollado hasta el presente (Gramsci citado en Rockwell, 1999). Sin embargo, es importante aclarar que Rockwell (1999), retomando los postulados de Vygotsky, resalta que las percepciones externas no se asimilan ni determinan enteramente el aprendizaje y/o el conocimiento de los individuos, sino que los individuos crean sus propios conocimientos y generan una conciencia particular conjuntando las percepciones de su contexto y sus conocimientos e ideas previas, sin embargo la pedagoga declara que las representaciones culturales de los individuos constituyen una parte importante del pensamiento crítico.
Rockwell (1999) declara que los educandos internalizan las herramientas y conocimientos percibidos en sus contextos sociales. Sin embargo, la pedagoga argumenta que el pensamiento crítico no es solamente una habilidad cognitiva abstracta, sino que para ella, es un asunto de un pensamiento real. El que la autora refiera que es un pensamiento real, no un tema cognitivo abstracto, quiere decir que se construye mediante la percepción y elaboración de la realidad por medio de los individuos involucrados, es decir, la conciencia crítica no es solamente un tema que los educadores presenten a los alumnos y estos tomen nota. Para que los educandos desarrollen una conciencia crítica es necesario que realicen prácticas y ejercicios que les permiten realizar estas elaboraciones con el contexto, de manera que ellos mismos, con la ayuda de un guía (que sería el educador), construyan esta conciencia.

De esta manera, en vez de considerar el pensamiento crítico como un proceso puramente cognitivo abstracto, Rockwell (1999) lo presenta como una práctica cultural, que se construye de manera particular dependiendo del contexto. Por esto mismo, la conciencia crítica no se puede encasillar solamente en un contexto de educación formal, sino que puede desarrollarse en espacios no-tradicionales, donde se representen o discutan interpretaciones alternativas de la realidad. Pero esta discusión lleva a cuestionarse: ¿Qué tipo de instrumento educativo o qué práctica puede lograr que los educandos construyan esta conciencia crítica? Una respuesta a este cuestionamiento puede ser el MUN.

En los MUN, una de las capacidades que más se desarrollan es la conciencia crítica y la toma de decisiones, esta aseveración se realiza ya que se ha realizado una investigación de tesis de maestría donde se analizan las capacidades fomentadas por los MUN, entre otros temas (Márquez Duarte, 2018), por lo que los postulados de la pedagogía crítica sostienen la importancia de ejercicios como el caso de estudio que se analiza en este documento. Así mismo, la dinámica de los MUN que es explicada a con- 
tinuación, tiene un componente constructivista en el centro.

Los Modelos de Naciones Unidas (MUN) se pueden definir como simulaciones para aprender las formas y objetivos de la cooperación internacional y la toma de decisiones a nivel internacional (Patterson, 1996). Por otro lado, si los MUN se analizan como una simulación política se pueden entender como un modelo de una parte de la realidad, generalmente de una situación de toma de decisiones y resolución de conflictos, con el objetivo de aprender sobre la interacción y procesos de la situación simulada (DiCamillo, \& Gradwell, 2013). Una tercera forma de entender a los MUN es como modelos de simulación; estos son una réplica o modelo operativo de la realidad, que busca recrear una situación. Al hacerlo, es posible conocer el proceso de elección de los actores involucrados y aprender de los procesos (Díaz, 2016; Patterson, 1996). Estos Modelos permiten a las y los participantes conocer realidades distintas a su realidad nacional, así mismo les permite contrastar problemas y soluciones posibles, posibilitando encontrar nuevos caminos para resolver las coyunturas internacionales sobre la base del diálogo, el consenso y la paz (HMUN, s/f).

Desde un enfoque educativo, los MUN aportan positivamente a la formación ciudadana en la escuela, haciendo una diferencia en cuanto al conocimiento de los asuntos públicos, desde nivel local hasta el internacional, sus valores y la motivación a ejercer la participación ciudadana, a esta formación también se le conoce como democratic schooling (Coviello, 2010; Print, 2007).

Para fines de este documento, se usa el siguiente concepto para los MUN basado en un trabajo académico previo (Márquez, 2017): eventos de "simulación" de Organismos Internacionales (OI) y/u órganos legislativos. En estos Modelos, los participantes representan al Embajador de un país u ONG o a un legislador, donde presentan una postura oficial (resultado de una investigación previa), con la cual, mediante el diálogo, debate, persuasión, se busca llegar a un proyecto de resolución, siendo la primera etapa una hoja de trabajo.

Como se percibe por la definición presentada, los participantes de estas simulaciones asumen un rol específico, con el cual se tienen que desempeñar a lo largo del MUN. Esta característica hace que los participantes tengan que investigar la información relevante para poder cumplir el rol asignado y puedan tener una experiencia de mayor aprendizaje dentro de la simulación. Este tipo de simulaciones tienen la particularidad de ser "de juego de rol". Las simulaciones de juego de rol hacen que los participantes se pongan en el lugar de otros y tengan una visión más amplia sobre las situaciones abordadas. Esto hace que desarrollen empatía por perspectivas e ideas alternativas, así como puntos de vista hasta entonces desconocidos, de esta manera los participantes aumentan su entendimiento y el de otros. El aspecto de los juegos de rol en los MUN ha sido resaltado en investigaciones previas, abonando a su utilidad como herramienta pedagógica (Agarwal, 2014; Chasek, 2005; Kempston, \& Thomas, 2014).

Un tema que es altamente practicado en los MUN es el proceso de cross-cultural understanding, así como la práctica del protocolo diplomático, incluyendo los procesos de los organismos simulados, estos temas generan un aprendizaje sumamente valioso para los participantes (Márquez Duarte, 2018; Obendorf, \& Randerson, 2012). La idea de cross-cultural understanding se refiere al entendimiento y empatía entre personas de diferentes culturas, factores que fomentan la tolerancia, la negociación y la cooperación. Dentro de esta lógica, el cross-cultural understanding va de la mano con la visión denominada de "ciudadanía global". La ciudadana global como enfoque, es aquel que consiste en crear una conciencia en los individuos de promover un cambio social, sin imponer una visión de cómo debe ser ese cambio, esto se alcanza mediante la creación de espacios seguros para analizar y experimentar otras formas de pensar en los otros y de interactuar con los otros. 
Se trata de empoderar individuos para tener una visión más integral e inclusiva del mundo, sin imponer una visión (de Andreotti, 2014). En un panorama tan internacionalizado, la ciudadanía global ha tomado una gran importancia dentro de la sociedad internacional, favoreciendo la formación y educación con visión global.

Así mismo, dentro de la educación ambiental, Rodríguez (2011) declara que los procesos de socialización en la escuela generan conciencia en los estudiantes sobre las relaciones del individuo y su contexto; de esta manera los MUN, por el rol de las interacciones sociales en el mismo, se convierte en una herramienta pedagógica constructivista muy útil en la educación ambiental.

\section{Metodología}

Como se ha desarrollado anteriormente, en el MUN, los participantes representan un rol específico, en este caso de estudio, los participantes fueron estudiantes de la maestría en educación; campo educación ambiental (MECEA) de la Universidad Pedagógica Nacional (UPN) Unidad 021 en Mexicali, Baja California, México y representaron al embajador de un país. A los estudiantes se les informó sobre el comité a simular y el tema con más de un mes de anticipación, así como de las reglas a utilizar en la simulación. Así mismo se les solicitó que entregaran un documento de postura oficial, con base en una investigación previa sobre el tema y el rol específico de cada uno de ellos.

El comité simulado fue la UNESCO (Organización de las Naciones Unidas para la Educación, la Ciencia y la Cultura), que es uno de los comités de la Organización de las Naciones Unidas (ONU). Al ser estudiantes de educación ambiental se eligió a la UNESCO ya que es el comité encargado de todos los temas educativos de la ONU, y para enfocar el ejercicio a su maestría el tema de la simulación fue: Relevancia de la educación ambiental en el marco de la agenda 2030 de la Organización de las Naciones Unidas. El grupo estuvo compuesto por siete estudiantes adultos que nunca habían participado en un MUN, y se fungió como moderador al ser el profesor del grupo.

Las reglas que se utilizaron en la simulación fueron las siguientes (vale la pena aclarar que las reglas pueden variar ligeramente de simulación en simulación, ya que este MUN fue realizado en clase, con un tiempo aproximado de dos horas y media en total, se adecuaron las reglas para su mejor desarrollo en el contexto específico del grupo): 1. Vestimenta formal o en su caso, vestimenta típica del país. 2. El lenguaje es formal y quedan prohibidas las palabras altisonantes. 3. Se prohíbe el uso de la 1ra. persona en singular, es decir, se prohíbe decir "yo opino", ya que se está representando a un país o delegación no a un solo individuo, la forma de lenguaje permitida puede ser "México considera que...", "Nosotros analizamos la situación...". 4. Para hacer el uso de la palabra se debe de levantar el placcard y esperar a que el moderador de comité le otorgue la palabra. 5. El tiempo límite por cada participación es de un minuto (máximo), en el caso de lectura de posturas es de dos minutos. 6 . Para comunicarse entre delegados se hace uso de las nota diplomáticas, no se permite la comunicación directa entre delegados, a excepción del caucus simple. 7. Existen dos tipos principales de procedimientos: Caucus moderado y caucus simple. En el caucus moderado, los participantes levantan su placcard para que el moderador otorgue la palabra. En el caucus simple los participantes pueden levantarse y dialogar con los demás participantes de manera más informal. 8 . Para aprobar una hoja de trabajo se realiza una votación, que se aprueba por mayoría simple. 9. Para aprobar un anteproyecto de resolución se realiza una votación, que se aprueba por mayoría calificada ( $2 / 3$ partes).

Al MUN se le otorgó una ponderación para la calificación de la materia impartida, de manera que se viera reflejado el trabajo y esfuerzo en sus calificaciones y que hubiera un incentivo mayor para la preparación de los estudiantes. Ya que se tenía una clase con el grupo a la semana, 
con una duración de aproximadamente 2 horas y 45 minutos, se dedicó una sesión completa a analizar y discutir dos artículos académicos sobre los MUN como herramienta pedagógica, para que los estudiantes entendieran la pertinencia del ejercicio en su formación, así como para que tuvieran una compresión más integral del mismo. En esa misma sesión se explicaron las reglas, que los estudiantes ya tenían por escrito desde un mes antes aproximadamente, así mismo, se repartieron los países que cada uno de ellos representaría en la simulación. Esta sesión se realizó tres semanas antes de la simulación. Dos semanas después de esa sesión se dedicó aproximadamente la mitad de una sesión (una hora y media) a aclarar dudas y a realizar un ensayo de la simulación para que estuvieran mejor preparados los educandos para el ejercicio. Los criterios que se tomaron en cuenta para la evaluación individual de los participantes del MUN fueron el documento de postura oficial, la cantidad de participaciones, la calidad de participantes y el comportamiento diplomático y con apego a las reglas.

Para realizar el análisis del caso de estudio se usó un modelo enteramente cualitativo de investigación, con un fuerte enfoque etnográfico (Creswell, 2003), usando los instrumentos de observación participativa (con el apoyo de un diario de campo), así como de entrevistas semidirectivas. Algunas de las entrevistas fueron realizadas presencialmente y otras fueron realizadas de manera escrita, enviadas y recibidas por correo electrónico, en fechas posteriores al MUN. De los siete educandos participantes, seis de ellos respondieron las preguntas de la entrevista.

Es importante argumentar que el tipo de estudio que se realizó es el de estudio de caso de subtipo clínico, ya que se analizan casos específicos en cantidad limitada de una situación acotada, este tipo de estudio se usa para analizar individuos de colectivos complejos (como los MUN), la validez recae en el estudio intensivo de los casos (Gomm citado en Márquez Duarte, 2018).

Vale la pena resaltar que tradicionalmente los MUN son realizados para estudiantes de edu- cación superior o media superior. Anteriormente se participó como organizador en MUN en ambos niveles, pero este caso de estudio constituyó el primer MUN realizado a nivel de posgrado, factor que brinda relevancia a la investigación y que ha determinado ciertos resultados de la investigación.

\section{Análisis y resultados del caso de estudio}

El MUN se realizó en una sesión, dedicada enteramente al ejercicio, con una duración de aproximadamente dos horas y 30 minutos.

Como primeras observaciones del ejercicio se percibió que los estudiantes se vistieron con ropa formal, todos leyeron su postura oficial; tuvieron buenas propuestas y la discusión fue diplomática y respetuosa. En cuanto a las percepciones de los participantes en general sobre el MUN, hubo opiniones variadas, sin embargo los temas generales que fueron mencionados por los estudiantes fueron relativos a llegar a acuerdos, a conocer otros puntos de vista, a trabajar de manera colectiva y a conocer los procesos de OI.

Uno de los participantes declara que:

Algo más que me pareció muy interesante fue las aportaciones de los compañeros, conocer un poco sobre la situación de China, de Brasil, de Alemania... me doy cuenta que la percepción de las personas es distinta" (Participante 1 , entrevista personal).

Lo sostenido por el participante 1 es relevante para esta investigación, ya que se traduce como el desarrollo de la capacidad de cross-cultural understanding, que ha sido discutida con anterioridad, $y$ es una capacidad valiosa para un educador, ya que el educador tiene que saber interactuar y formar a estudiantes de diferentes culturas al mismo tiempo, al mismo tiempo que se genera empatía. Respecto al tema de llegar a acuerdos y trabajar de manera colectiva se declaró lo siguiente:

...Sentía que algunos compañeros iban a tener disgustos, pero al parecer no fue así, todo salió 
muy bien, y considero que aprendí mucho, en cómo ponernos de acuerdo para llegar a un fin en beneficio de todos" (Participante 2, entrevista personal).

En este caso, la participante 2 resalta la cooperación que se desarrolló por los participantes, de una manera diplomática, buscando que se tomaran decisiones para un beneficio colectivo, sin imposición de ideas, mediante las interacciones sociales, como se ha analizado anteriormente con los postulados constructivistas, especialmente de Vygotsky. El poder dialogar y tomar decisiones de manera conjunta son capacidades sumamente valiosas a nivel personal y a nivel profesional, sobre todo como educador, ya que al estar frente a grupo es necesario saber mediar y lograr un ambiente con los menos conflictos posibles.

Uno de los cuestionamientos que se realizó a los participantes fue sobre los conceptos y/o conocimientos que pusieron en práctica en el MUN, ya que al estar en la segunda mitad de la maestría en educación ambiental, uno de los objetivos principales era que pusieran en práctica los conocimientos obtenidos en las clases cursadas, en temas relativos a la educación ambiental. En este tema los participantes declararon que pusieron en práctica sus conocimientos y preparación sobre educación ambiental, impacto ambiental, cooperación internacional, la agenda 2030, diálogo y protocolos/reglas que se siguen en OI y cumbres internacionales. Sobre este cuestionamiento, es importante resaltar que el diálogo (negociación y tolerancia), así como el seguir reglas/protocolos (disciplina) son consideradas como capacidades que se pueden desarrollar por participar en MUN, que se diferencian de los conocimientos, tomando en cuenta que las capacidades se definen de la siguiente manera: "La capacidad de una persona refleja las combinaciones alternativas que la persona puede alcanzar, y de la cual él o ella puede escoger un conjunto" (Sen, 1993, p. 31). Es decir, las capacidades son "la combinación de alternativas de las realizaciones que un individuo puede alcanzar para lograr su bienestar y alcanzar el desarrollo deseado" (Márquez Duarte, 2018, p. 50).

Uno de los aspectos más importantes sobre el MUN para analizar su utilidad y efectividad como instrumento pedagógico con un enfoque constructivista es saber qué capacidades fomenta en los participantes. En una investigación previa (Márquez Duarte, 2018) se prueba que el MUN fomenta una serie de capacidades en sus participantes, por lo que resultó pertinente saber qué capacidades fomenta en el caso específico de esta investigación (estudiantes de la MECEA). En la investigación previa citada se obtuvieron un total de 19 capacidades que los MUN pueden fomentar, estas 19 capacidades fueron las señaladas por los individuos entrevistados (18 estudiantes o graduados universitarios). Ya que el caso de estudio de la presente investigación es de un grupo de estudiantes adultos de posgrado, se seleccionaron 15 capacidades de las que los participantes podían seleccionar las que consideran que les fomentó el MUN. En la siguiente tabla se presentan las capacidades señaladas por los estudiantes de la MECEA:

Tabla 1. Capacidades fomentadas por los alumnos en estudiantes de la MECEA

\begin{tabular}{|l|c|l|c|l|}
\hline \multicolumn{1}{|c|}{ Capacidades } & Frecuencia & \multicolumn{1}{|c|}{ Capacidades } & Frecuencia & \multicolumn{1}{|c|}{ Capacidades } \\
\hline Hablar en público & 5 & Organización & 2 & Investigación \\
\hline Negociación & 4 & Disciplina & 4 & $\begin{array}{l}\text { Cross-cultural } \\
\text { understanding }\end{array}$ \\
\hline Trabajo en equipo & 4 & Debate & 5 & Auto-confianza \\
\hline Tolerancia & 3 & Lenguaje formal & 6 & Toma de decisiones \\
\hline Trabajar bajo presión & 2 & $\begin{array}{l}\text { Redacción de do- } \\
\text { cumentos formales }\end{array}$ & 1 & Empatía \\
\hline
\end{tabular}

Fuente: Elaboración propia con resultados del trabajo de campo. 
Las capacidades más fomentadas según los estudiantes de la MECEA son: el lenguaje formal, seguida de la capacidad de debate, así como hablar en público. Sobre la capacidad de hablar en público, por ejemplo, uno de los estudiantes señala:

Para hablar en público, en mi opinión, se requiere conocimientos previos, organización, disciplina labor de búsqueda y lenguaje formal" (Participante 3, entrevista personal).

El alumno declara que el MUN le fomentó la capacidad de hablar en público, y que a su vez esta capacidad requiere de la capacidad de investigación, lenguaje formal, disciplina y organización, por lo que este fomento de capacidades fue transversal, no de manera aislada entre una u otra capacidad.

Un importante aspecto sobre los MUN es la interacción de los participantes, debido a este se les preguntó a los estudiantes de la MECEA si consideraban que la interacción con los demás participantes en el MUN tuvo efectos en su experiencia, señalando la razón de su respuesta. Cabe resaltar que todos los entrevistados declararon que la interacción en el MUN tuvo efectos en su experiencia como participantes. Algunas de las respuestas de los estudiantes sobre el efecto de las interacciones fueron:

Sí. El ejercicio permite ver no solo desde la propia perspectiva, sino también desde otras perspectivas" (Participante 4, entrevista personal). "Para realizar el ejercicio requirió la colaboración de casi todos los participantes; esto nos condujo a trabajar en equipo y con actitud proactiva" (Participante 3 , entrevista personal). "Sí, ya que me ayudó a escuchar las distintas posturas y situaciones que las demás naciones viven" (Participante 5, entrevista personal).

Lo referido por los estudiantes sobre la interacción es relevante para la investigación, ya que evidencia el enfoque constructivista de los MUN; como se discutió en el estado de la cuestión, la solución de problemáticas de manera colectiva, con un guía (en este caso el moderador) es un ejemplo claro de los postulados constructivistas, especialmente de Vygotsky. Uno de estos postulados es la ZDP, misma que se discutió anteriormente y que se materializa en la dinámica de simulación de los MUN, donde los mismos educandos mediante el diálogo, debate y trabajo en equipo llegan a plantear soluciones a la problemática definida, siempre con un guía (moderador). Así mismo, al tocarse temas como el generar empatía, cross-cultural understanding y trabajo en equipo, que son capacidades útiles y requeridas como ser humano, así como educador, los MUN se constituyen como herramientas pedagógicos relevantes, especialmente como educador ambiental. Al respecto se considera importante resaltar el enfoque de interacción simbólica (Goffman, 2006), ya que se puede usar como base para analizar los cambios que los MUN generan al modificar comportamientos (Márquez Duarte, 2018). Este enfoque se explica como el rol o papel que adoptamos cada uno de nosotros como individuos en determinados escenarios y/o situaciones; en el MUN se asume el rol específico de delegado, mismo que determina comportamientos, conocimientos y habilidades desarrolladas en el ejercicio y que, como lo establece Goffman, se interiorizan en el individuo aunque el MUN acabe.

Un último cuestionamiento realizado a los estudiantes de la MECEA fue si cambiarían algún aspecto del MUN para que les generara mayor aprendizaje/utilidad. En este punto, la mayoría de los entrevistados señalaron que lo que cambiarían es el tiempo de preparación y los ensayos previos; declararon que les hubiera gustado tener más sesiones y más tiempo para ensayar el MUN y prepararse mejor para su participación en el mismo.

Vale la pena aclarar que se considera (con los resultados obtenidos en la investigación) que los MUN pueden funcionar como un ejercicio articulador de una formación adecuada para educadores, en este caso ambientales. Para sostener este argumento, el informe Delors (1996) 
presenta postulados interesantes; el informe resalta los cuatro pilares de la educación: aprender a conocer, aprender a ser, aprender a hacer y aprender a convivir. De los cuatro pilares, el pilar de aprender a vivir con otros/convivir es de especial relevancia para esta investigación. En este pilar, se busca una educación que permita solucionar los conflictos de manera pacífica y armónica, fomentando el conocimiento de los demás. Esto es difícil de alcanzar ya que una gran cantidad de individuos tienden a menospreciar y tener prejuicios contra los que son diferentes a ellos, reaccionando con agresión al "otro", como resultado del desconocimiento y el miedo hacia ese "otro". En el MUN se fomenta el cross-cultural understanding como discutido anteriormente; esta capacidad busca la empatía y convivencia armónica para evitar y resolver conflictos, por lo que ayuda a la tarea de los educadores, especialmente los pilares señalados por Delors.

\section{Conclusiones}

El MUN realizado con estudiantes de la MECEA ha sido un ejercicio pedagógico con enfoque constructivista que ha permitido estudiar un caso específico de su aplicación como instrumento para generar aprendizaje y, sobre todo, desarrollo de capacidades en sus participantes. Aunque los MUN han sido realizados como ejercicio educativo con estudiantes desde hace ya algunas décadas, comúnmente se realizan con estudiantes de nivel medio superior $y$ superior, por lo que el caso estudiado, al ser de un grupo de estudiantes de posgrado, constituye un caso peculiar e interesante que arroja indicios y conclusiones que aportan al conocimiento en el campo de la educación.

Como se pudo apreciar en el apartado anterior, el trabajo de campo mostró que los estudiantes declararon haber obtenido aprendizajes de conocimientos relacionados a temas de su programa de estudios (educación ambiental), así como de la misma dinámica del MUN, que como se discutió anteriormente tiene un fuerte enfoque constructivista. Más allá de los conocimientos, es sumamente valioso el desarrollo de capacidades que los estudiantes declaran haber obtenido en el MUN. De entre las capacidades más desarrolladas por los estudiantes de la MECEA se encuentran el lenguaje formal, el debate, hablar en público, la negociación, la empatía y el trabajo en equipo.

Como se puede apreciar, el desarrollar una conciencia y ciudadanía global e intercultural, las interacciones entre los individuos y su contexto, las soluciones a problemas de manera colectiva, así como el transformarse en agentes de cambio son puntos principales que se trabajan en los MUN y que han sido abordados en este trabajo. Así mismo, como se ha discutido anteriormente, los MUN son benéficos por el fomento de capacidades como cross-cultural understanding, ciudadanía global, empatía, diálogo, negociación, entre otras, que como se analiza en el caso de estudio de esta investigación, son sumamente importantes para un educador, y especialmente para un educador ambiental, ya que lo fomentado por los MUNs se encuentra en la línea de los valores ambientales que representan la esencia de la educación ambiental, como el diálogo, la pluralidad, la empatía y la convivencia (Aguirre Ibarra, \& Mata González, 2011; Rodríguez Pinson, 2011).

En esta misma línea, vale la pena resaltar la utilidad de los MUN como herramienta de integración de interacciones sociales entre individuos en la escuela, así como de desarrollo de conciencia crítica. Sobre este tema es importante retomar los postulados de Freire analizados en el estado de la cuestión: Los MUN al ser una herramienta pedagógica donde se desarrolla una conciencia y capacidad crítica en los individuos a través del diálogo, el debate y la negociación, permite a los educandos que generen su propio conocimiento y entendimiento de las situaciones sociales, políticas y económicas planteadas, de manera que los MUN se materializan como un método "activo" (como lo planteaba Freire), donde los alumnos desarrollen su propia con- 
ciencia mediante situaciones que se plantean por el educador, sin ser impuestos los conocimientos o el punto de vista específico sobre el contexto o la situación del educador, ya que el educador solamente funge como moderador, permitiendo que el MUN se transforme en un espacio donde los mismos alumnos generan conciencia, capacidades y conocimientos desde su interacción y su propio entendimiento, llegando a reflexiones más integrales e inclusivas, y constituyéndose como un método que coadyuve a que los participantes se transformen en agentes de cambio.

Otro de las consideraciones en la conclusión de este trabajo es recapitular la importancia del juego de rol en el MUN y la ZDP desde un enfoque constructivista; como se puede apreciar en el apartado de trabajo de campo, el hecho de que los participantes hayan tenido que representar roles específicos (Embajador de un país) e interactuar con los demás que también tuvieron roles generó conocimientos y aprendizajes positivos en los estudiantes, sosteniendo la discusión presentada en el apartado anterior sobre cómo el MUN es una herramienta constructivista, siguiendo los postulados tanto de Piaget, como de Vygotsky, analizados en el estado de la cuestión.

Como capitulaciones finales sobre el MUN, es de resaltar positivamente que en este caso de estudio, aunque el MUN durara solamente 2 horas y media (y un receso de 15 minutos), se pudo llegar a negociar y redactar una hoja de trabajo, que es el borrador de un documento de proyecto de resolución, llamado así ya que en la ONU se llegan a proyectos de resolución para atacar los problemas en la comunidad internacional. Un claro ejemplo de proyecto de resolución es la Declaración como resultado de la Cumbre de Desarrollo Sostenible de la ONU, de la cual surgieron los 17 Objetivos de Desarrollo Sostenible (ODS) en 2015.

Es importante aclarar que esta investigación ha sido un caso de estudio específico, por lo que no se deben generalizar los resultados para aplicarse a cualquier caso de estudio. Sin embar- go, esta investigación sirve como base para realizar más investigaciones con más casos de estudio y mayores recursos (materiales, humanos y de tiempo), con el objetivo de seguir analizando los MUNs como método pedagógico, desde un enfoque constructivista y de pedagogía crítica.

\section{Referencias bibliográficas}

Agarwal, J. K. (2014). Impact of Model United Nations Conferences on India's Youth. OIDA International Journal of Sustainable Development, 7(8), 6. (https://bit.ly/2TUhVZu).

Aguirre Ibarra, M. C., \& Mata González, E. (2011). La ética: Punto de encuentro de la educación ambiental y el ser profesional. Espiral, Revista de Docencia e Investigación, 1(1), 55-66. https://doi.org/10.15332/erdi.v1i1.106

Chasek, P. S. (2005). Power Politics, Diplomacy and Role Playing: Simulating the UN Security Council's Response to Terrorism. International Studies Perspectives, 6(1), 1-19. https://doi.org/10.1111/j.1528-3577.2005.00190.x

Coviello, A. (2010). When standardized test success represents survival: Creating opportunities for democratic participatory development in class 5-340 (PhD). Columbia University, New York. (https://bit.ly/2FwEgsM).

Creswell, J. W. (2003). Research design: Qualitative, quantitative, and mixed methods approaches (Vol. 4). CA: Sage Thousand Oaks.

de Andreotti, V. O. (2014). Soft versus critical global citizenship education. Development education in policy and practice 3(1), 40-51. (https://bit.ly/2vMkGng).

Delors, J. (1996). Los cuatro pilares de la educación. En J. Delors (Ed.), La educación encierra un tesoro (pp. 91-103). Santillana. UNESCO.

Díaz, U. D. (2016). Modelos de Naciones Unidas: una herramienta para la cultura de paz (Licenciatura). Ciudad de México: Universidad Nacional Autónoma de México.

DiCamillo, L., \& Gradwell, J. M. (2013). To Simulate or Not To Simulate? Investigating Myths about Social Studies Simulations. The Social Studies, 104(4), 155-160. https://doi.org/10.1080/00377996.2012.716094 
Freire, P. (1970). Pedagogia do oprimido (Vol. 21). Paz e Terra.

Goffman, E. (2006). The presentation of self. Life as theater: A dramaturgical sourcebook. Penguim Random House.

HMUN (s/f). HMUN: About Us. (https://bit.ly/2HhGfmn) (20-11-2016).

Kempston, T., \& Thomas, N. (2014). The Drama of International Relations: A South China Sea Simulation. International Studies Perspectives, 15(4), 459-476.

https://doi.org/10.1111/insp.12045

Márquez, F. (2017). Los Modelos de Naciones Unidas, su cooperación trasnacional y la participación ciudadana juvenil. Muuch' Xiímbal: Caminemos Juntos, (4), 193-226.

(https://bit.ly/2FvKd9l).

Márquez Duarte, F. (2018). Participación ciudadana juvenil en Baja California, México y California, Estados Unidos: el caso de los Modelos de Naciones Unidas. (M.A.). México: El Colegio de la Frontera Norte, Tijuana, B.C.

Mata González, E., \& Aguirre Ibarra, M. C. (2007). Desarrollo sustentable actual: una reconsideración desde la educación ambiental. Espíritu científico en acción, 3(6), 26-34.
Obendorf, S., \& Randerson, C. (2012). The Model United Nations simulation and the student as producer agenda. Enhancing Learning in the Social Sciences, 4(3). (https://bit.ly/2Mf4Jvz).

Patterson, J. R. (1996). Model United Nations simulations: An inquiry into active learning, roleplaying, and role identification as they impact participants' sense of political efficacy (Ph.D.). USA: Ann Arbor. (https://bit.ly/2RX0ud8).

Print, M. (2007). Citizenship Education and Youth Participation in Democracy. British Journal of Educational Studies, 55(3), 325-345. https://doi.org/10.1111/j.1467-8527.2007.00382.x

Rockwell, E. (1999). Historical Consciousness and Critical Thinking: Reflections on an Indigenous Movement. The Journal of Educational Foundations, 13(3), 23.

Rodríguez Pinson, M. C. (2011). Hacia una construcción de ciudadanía ambiental en la escuela. Espiral, Revista de Docencia e Investigación, 1(1), 13-20. https://doi.org/10.15332/erdi.v1i1.102

Schunk, D. H. (1997). Teorías del aprendizaje (6a ed.). Pearson Educación.

Sen, A. (1993). Capability and Well-Being. En M. Nussbaum \& A. Sen (Eds.), The quality of life (p. 30). Oxford: Clarendon Press.

(https://bit.ly/2VT0p9L). 